\title{
ARTICLE
}

\section{Non-autonomous DAF-16/FOXO activity antagonizes age-related loss of $C$. elegans germline stem/progenitor cells}

\author{
Zhao Qin ${ }^{1} \&$ E. Jane Albert Hubbard ${ }^{1}$
}

\begin{abstract}
Stem cells maintain tissues and organs over the lifespan of individuals. How aging influences this process is unclear. Here we investigate the effects of aging on $C$. elegans germline stem/progenitor cells and show that the progenitor pool is depleted over time in a manner dependent on inhibition of DAF-16/FOXO by insulin/IGF-1 signalling (IIS). Our data indicate that DAF-16/FOXO activity in certain somatic gonad cells is required for germline progenitor maintenance, and that this role is separable from the effect of DAF-16/FOXO on organismal aging. In addition, blocking germ cell flux, similar to reducing IIS, maintains germline progenitors. This effect is partially dependent on gonadal DAF-16/FOXO activity. Our results imply that (1) longevity pathways can regulate aging stem cells through anatomically separable mechanisms, (2) stem cell maintenance is not necessarily prioritized and (3) stem cell regulation can occur at the level of an entire organ system such as the reproductive system.
\end{abstract}

\footnotetext{
${ }^{1}$ Departments of Cell Biology and Pathology, The Helen L. and Martin S. Kimmel Center for Stem Cell Biology, Skirball Institute for Biomolecular Medicine, New York University School of Medicine, 540 First Avenue, New York, New York, USA. Correspondence and requests for materials should be addressed to E.J.A.H. (email: jane.hubbard@med.nyu.edu).
} 
S tem cells are remarkable for their ability to maintain adult tissue homeostasis and to respond to injury. With age, however, stem cells experience numerical and/or functional decline or changes in differentiation potential, which can lead to bias in cancer predisposition, tissue degeneration and increased susceptibility to tissue damage ${ }^{1}$. Therefore, a better understanding of how aging affects stem cells may provide important insights relevant to age-related diseases and stem cell-based therapy.

The nematode $C$. elegans provides an attractive model for studying stem cell aging. First, C. elegans possesses a relatively simple and accessible stem cell system - the germline stem cellsthat, similar to stem cell systems in other organisms, uses the conserved Notch signalling pathway as the major pathway to maintain stem cell fate ${ }^{2,3}$ (Fig. 1a). Second, C. elegans is a wellestablished genetic model for aging. The relatively short (2-3 weeks) lifespan of laboratory worms facilitates the analysis of age-

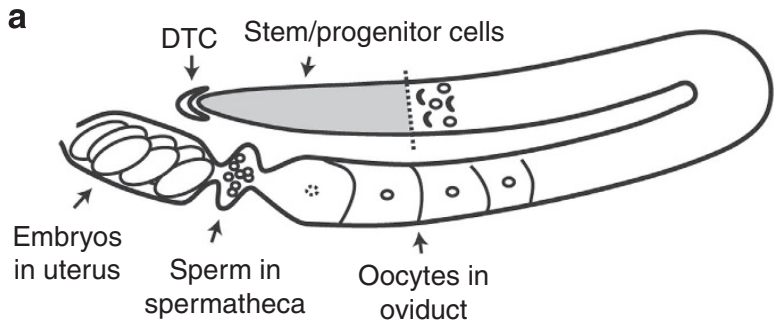

b

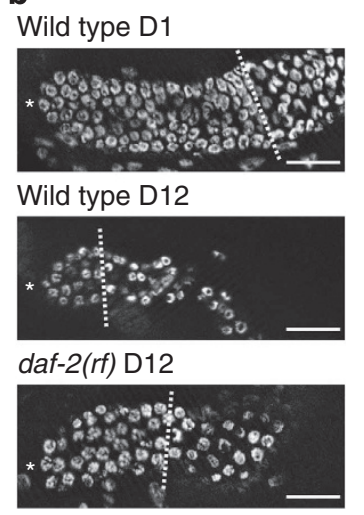

C

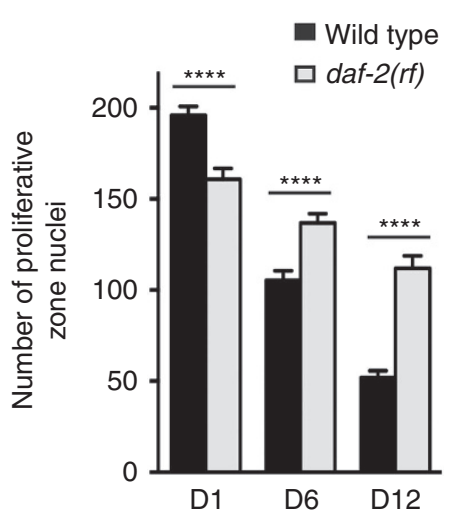

Figure 1 | The DAF-2/insulin-IGF-like receptor (IIR) promotes age-related loss of germline progenitor cells. (a) Schematic drawing of the adult $C$. elegans hermaphrodite germ line. Stem/progenitor cells (grey shading) are located in the distal region of the germ line that is capped by the distal tip cell (DTC), the stem cell niche. As germ cells divide and move proximally, they enter meiosis. Nuclei in early stages of prophase of meiosis I (leptotene and zygotene) are crescent shaped. By convention, the appearance of two or more crescents in a full ring of nuclei marks the proximal border of the progenitor pool ${ }^{37}$ (dotted line). Germ cells eventually differentiate into sperm that are stored in the spermatheca followed by oocytes that reside in the adult oviduct. In response to signals from the sperm, oocytes mature one by one and are fertilized as they pass through the spermatheca. (b) Representative DAPI-stained wild-type and daf-2(rf) germ lines. Asterisk indicates the distal end of the germ line, and the dotted line indicates the proximal boarder of the proliferative zone.

Scale bars, $20 \mu \mathrm{m}$. (c) Time course of germline progenitor depletion in wildtype and daf-2(rf) animals. Note that daf-2(rf) mutants start with fewer germline progenitor cells than wild type on adult day 1 (D1) (see text for details). Error bar indicates s.e.m.; ${ }^{\star \star \star \star} P<0.0001$ by two-tailed Student's $t$-test; also, pairwise comparisons within genotypes for each time point $P<0.0001$ by Student's $t$-test. Allele used: daf-2(e1370). See Supplementary Table 3 for complete data. DAPI, 4,6-diamidino-2phenylindole. dependent events. Many of the longevity pathways initially identified in worms are highly conserved for aging functions across species 4 .

Here we investigated the effects of age on the pool of undifferentiated proliferative cells in the distal end of the $C$. elegans germ line. This population of cells includes both germline stem cells and their proliferative progeny (hereafter referred to as 'germline progenitor cells', as no markers currently distinguish stem cells from their proliferative progeny; Fig. 1a). We observed a marked age-dependent decline in the number of germline progenitor cells that is far less severe in mutants with reduced insulin/insulin-like growth factor-1 (IGF-1) signalling (IIS). In addition, we found that DAF-16/FOXO acts downstream of IIS to regulate germline progenitor maintenance. By modulating DAF$16 / F O X O$ activity in a tissue-specific manner, we found that the extent of germline progenitor loss over time could be uncoupled from the rate of organismal aging. Surprisingly, DAF-16/FOXO activity is not required in germ cells, but rather is required in somatic cells of the gonad to prevent germline progenitor cell loss. Specifically, DAF-16/FOXO activity is required at the proximal end of the reproductive tract, in cells that contact transiting gametes and embryos. Finally, we determined that germ cell flux also influences germline progenitor maintenance through DAF-16/FOXO-dependent and DAF-16/FOXO-independent mechanisms.

\section{Results}

The number of germline progenitors decreases with age. We determined the number of progenitor cells in the germline proliferative zone of wild-type $C$. elegans hermaphrodites during adulthood and found that the pool of germline progenitors decreased markedly over time. Under normal laboratory growth conditions, germline progenitors accumulate during larval development to a pool of $\sim 200-250$ cells at early adulthood ${ }^{5}$. This pool is maintained for $24-36 \mathrm{~h}$, but decreases thereafter ${ }^{5-7}$. We found that by 12 days of adulthood, the progenitor pool was reduced to $\sim 50$ cells in the wild type (Fig. 1 b,c).

We considered three possible cellular mechanisms for the loss of germline progenitors in aged worms as follows: (1) cell death, (2) a cell cycle defect and/or (3) a change in the balance between proliferation (mitosis) and differentiation (meiosis), such that meiotic entry occurred more distally in older animals. For cell death, we examined the ced-3/Caspase mutant, as germ cell death in C. elegans is dependent on the core apoptotic machinery ${ }^{8}$. We found that although clusters of extra germ cells accumulated in the pachytene stage in old ced-3(-) animals (Supplementary Fig. 1a), the number of germline progenitor cells was not significantly altered (Supplementary Fig. 1b), suggesting that inappropriate progenitor apoptosis does not account for germline progenitor loss over time. To assess cell cycle, we measured the mitotic index in the proliferative zone and found that the mitotic index of day 12 (D12) animals was reduced relative to that of D1 animals (Supplementary Fig. 1c), suggesting a role for cell cycle in progenitor loss. For the mitosis versus meiosis cell fate decision, we determined the distance from the distal tip to the proximal border of the proliferative zone as a measure of the effective 'reach' of the distal tip cell signal to deter meiotic entry. We found that the distance to meiotic entry was reduced in D12 animals compared with D1 animals (Supplementary Fig. 1d), indicative of an additional role for cell fate decision in this phenotype. Notch signalling mediated by the Notch family receptor GLP-1 controls the cell fate decision in the $C$. elegans germ line ${ }^{2,9}$. Consistent with a prominent cell fate role, the combination of aging and reduced $g l p-1$ activity enhanced the penetrance of the 'all meiotic' Glp-1 phenotype (in which all germ cells enter meiosis and the 
progenitor pool is lost). For example, in contrast to the wild type that retained $\sim 100$ progenitor cells on D6, 30\% of $g l p-1$ reduction-of-function ( $r f$ ) mutant animals had the 'all meiotic' phenotype. By D12, nearly all $g l p-1(r f)$ animals failed to maintain a progenitor pool (Supplementary Fig. 1e). Together, we conclude that both reduced cell division rate and altered cell fate, but not cell death, contribute to the loss of germline progenitors over time.

\section{DAF-2/IIR promotes age-related loss of germline progenitors.} Because the germline progenitor cell loss phenotype is age related, we asked whether genes that affect the rate of aging would influence germline progenitor maintenance over time. Reducing the highly conserved IIS delays aging and extends lifespan in many organisms ${ }^{4}$. Worms with reduced activity of the sole insulin/IGF-like receptor (IIR) in C. elegans, daf-2, live twice as long as wild type ${ }^{10}$. In addition, some daf-2(rf) mutants have extended reproductive span and produce progeny late in life ${ }^{11,12}$. Therefore, we extended previous studies 7 and examined the D1-D12 time course of germline progenitor cell depletion in two daf-2(rf) mutants, daf-2(e1370) and daf-2(e1368). Due to a requirement for IIS in the accumulation of larval germline progenitors, daf-2(rf) animals start adulthood with fewer germline progenitors ${ }^{13}$ (D1, Fig. 1c and Supplementary Fig. 2). Despite this initial shortfall, both mutants maintained more progenitor cells on D6 and D12 compared with wild type (Fig. 1b,c; Supplementary Fig. 2). The maintenance is more pronounced in daf-2(e1370) in which D12 animals retained an average of twice as many progenitors as wild type $(112.0 \pm 6.8$ versus $52.0 \pm 3.7)$, and this allele was used in further analysis. We conclude that DAF-2/IIR signalling promotes age-related loss of germline progenitors.

DAF-16/FOXO acts downstream of DAF-2/IIR. IIS controls many processes in C. elegans, including metabolism, immunity, development and aging. In most cases, activation of the receptor, DAF-2, initiates a conserved kinase cascade that ultimately inhibits the FOXO transcription factor DAF-16 (ref. 14). To determine whether daf-16 mediates the effect of IIS on germline progenitor cell maintenance, we asked whether, similar to other phenotypic scenarios, the effect of reduced daf-2 activity could be suppressed by the loss of daf-16. We found that while loss of daf-16 alone did not affect germline progenitor loss over time compared with wild type (Fig. 2a, daf-16(-) versus wild type), in the absence of daf-16, daf-2(rf) animals lost their germline progenitors to the same extent as the wild type (Fig. 2a, daf-16(-); daf-2(rf) versus daf-2(rf) and wild type, respectively). Therefore, daf-16(+) activity is required for the ability of daf-2(rf) to maintain germline progenitors over time.

DAF-16/FOXO acts in the proximal somatic gonad. To determine whether daf-16(+) maintains germline progenitors in a germline-autonomous manner, we tested the effect of knocking down daf-16 by RNA interference (RNAi) in the daf-2(rf) background in the presence and absence of $r r f-1$. In the absence of $r r f-1$, RNAi effectiveness is reduced in the soma but retained in the germ line ${ }^{15,16}$. We found that while daf-2(rf) animals subject to daf-16 RNAi resembled the daf-16(-); daf-2(rf) double mutants and lost their germline progenitors similarly to wild type, this effect was not observed in the $r r f-1(-)$; daf-2(rf) animals subject to daf-16 RNAi (Fig. 2b). However, daf-16 RNAi was indeed effective in the $\operatorname{rrf}-1(-)$; daf-2(rf) background, as evidenced by suppression of the germline-autonomous larval germline proliferation phenotype caused by the daf-2(rf) mutation (Supplementary Fig. 3), consistent with previous results ${ }^{13}$. These results suggest that daf-16(+) is not required in the germ line for daf-2(rf) to preserve germline progenitors over time.

To determine the anatomical focus of daf-16(+) activity for germline progenitor maintenance, we employed two strategies as follows: (1) tissue-specific expression of a rescuing daf-16(+) transgene and (2) tissue-specific daf-16 RNAi. Surprisingly, we found that daf-16(+) expression in a subset of somatic cells in the proximal gonad is sufficient to maintain germline progenitors. First, expression of a $g f p:: d a f-16$ transgene from the fos-1a promoter in a subset of adult spermathecal and uterine cells ${ }^{17}$ (Supplementary Fig. 4a) restored the number of D12 progenitors in the daf-16(-); daf-2(rf) double mutant background to the level seen in daf-16(+); daf-2(rf) animals and to the level seen in daf-16(-); daf-2(rf) animals expressing the same gfp::daf-16 transgene from the daf-16 endogenous promoter (Fig. 2c,d). By contrast, expression of the same gfp::daf-16 transgene in the intestine, muscles or neurons did not affect germline progenitor cell maintenance (Fig. 2c). We verified both daf-16(+) activity by rescue of relevant phenotypes and the corresponding tissue-specific daf-16 transgene expression in aged animals, as previously reported ${ }^{18}$. The same negative result was observed with a daf-16::gfp transgene expressed in the distal tip cell $^{13}$, the stem cell niche (Supplementary Fig. 5a).

We confirmed the proximal somatic gonad requirement for daf-16(+) in germline progenitor maintenance by tissue-specific daf-16 RNAi. To achieve proximal somatic gonad-specific RNAi, we performed RNAi knockdown in the rde-1( - ) RNAi-deficient animals expressing $r d e-1(+)$ from the fos- $1 a$ promoter ${ }^{19}$. We found that knocking down daf-16 by proximal somatic gonad-directed RNAi resembled daf-16 RNAi in the whole animal, causing more pronounced germline progenitor depletion in the daf-2(rf) background (Fig. 2e).

Since expression of $g p:: d a f-16$ in the intestine has a marked effect on lifespan ${ }^{18}$, failure of the same transgene to influence germline progenitor cell maintenance (Fig. $2 c$ ) suggests that DAF16/FOXO regulates lifespan and germline progenitor maintenance separately. Another tissue that has been implicated in lifespan regulation is the hypodermis ${ }^{20}$. To test whether hypodermal IIS influences germline progenitor maintenance, we performed daf-2 RNAi in $r d e-1(-)$ animals expressing $r d e-1(+)$ from a hypodermis-specific version of the lin-26 promoter ${ }^{21}$. We found that hypodermis-specific daf-2 knockdown had no effect on progenitor maintenance, although daf-2 RNAi in the whole animal or in the proximal somatic gonad alone could mimic the effect of the daf-2(rf) mutation, maintaining more germline progenitors than the RNAi control on D12 (Supplementary Fig. 5b,c).

To further test the hypothesis that lifespan and germline progenitor cell maintenance are independent phenotypes that can be uncoupled anatomically, we asked whether the same proximal somatic gonad-expressing $g f p:: d a f-16$ transgene that was sufficient for germline progenitor maintenance would influence lifespan. We found a minimal difference between the lifespans of proximal somatic gonad daf-16(+) animals and their non-transgenic siblings: $16.1 \pm 0.5$ days and $14.8 \pm 0.4$ days, respectively $(P=0.04$; Supplementary Fig. $4 b)$.

Together, our data reveal a novel cell non-autonomous DAF-16/FOXO activity that is required for adult germline progenitor maintenance and that is anatomically separable from its previously described roles in lifespan regulation and other biological processes in C. elegans.

Germ cell flux promotes germline progenitor cell loss. The proximal somatic gonad is a region of the reproductive tract that contacts transiting gametes and embryos. Our finding that 

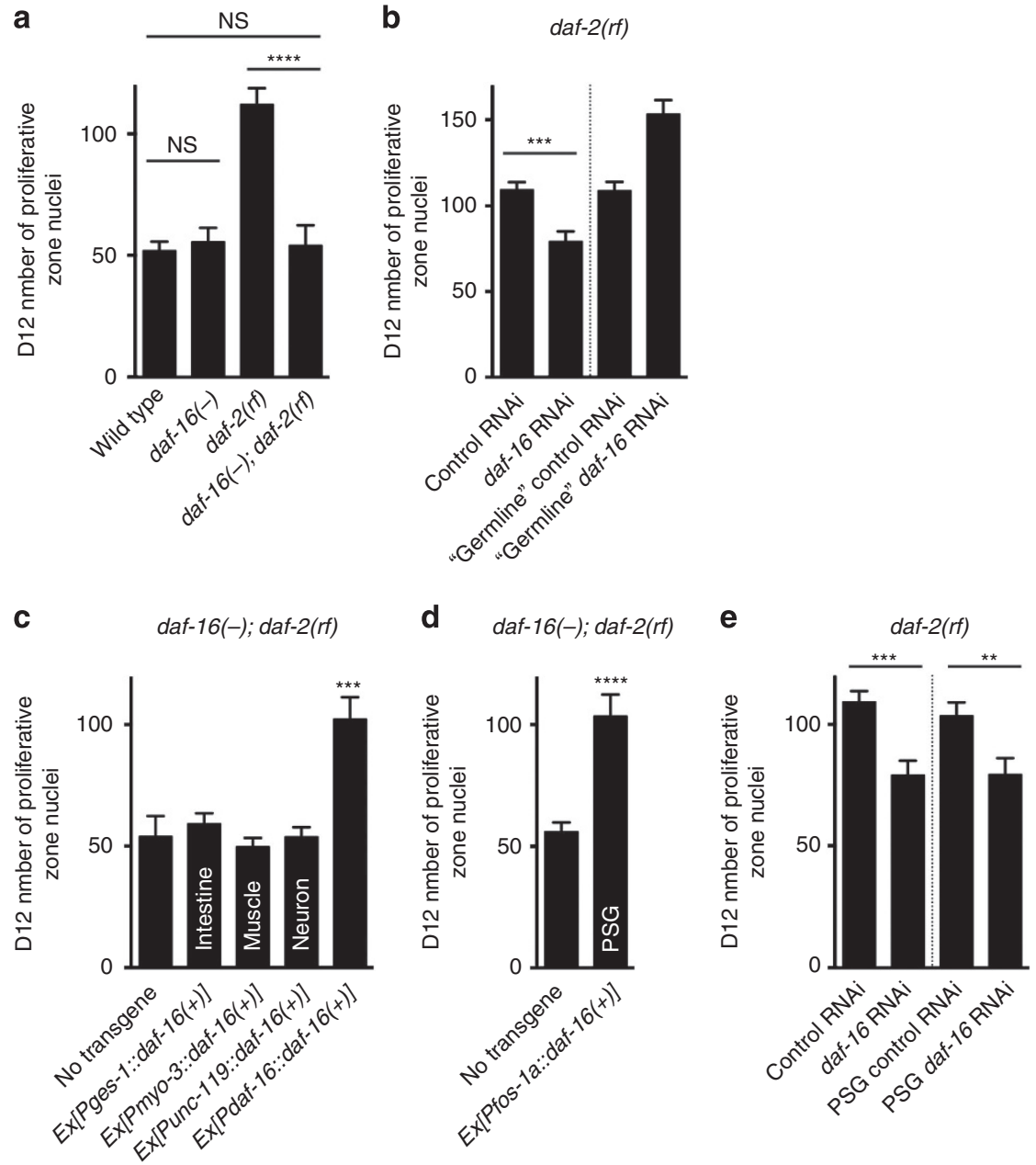

Figure 2 | Proximal somatic gonad (PSG) DAF-16/FOXO activity maintains germline progenitors. Average number of proliferative zone nuclei in D12 (a) wild-type, daf-16( - ), daf-2(rf) and daf-16( - ); daf-2(rf) animals, (b) daf-2(rf) and rrf- $1(-)$; daf-2(rf) animals treated with control and daf-16 RNAi, (c) daf-16( - ); daf-2(rf) and daf-16( - ); daf-2(rf) animals carrying a transgene expressing gfp::daf-16(+) from the ges-1, myo-3, unc-119 and daf-16 promoters, respectively, (d) daf-16( -$)$; daf-2(rf) and daf-16( - ); daf-2(rf) animals carrying a transgene expressing $g f p:: d a f-16(+)$ from the fos-1a promoter, (e) daf-2(rf) and daf-2(rf); rde-1(-); Is[Pfos-1a::rde-1(+)] animals treated with control and daf-16 RNAi. (b) Note that due to the germlineautonomous requirement for daf-2 in larval progenitor accumulation (which is daf-16 dependent), 'germline' daf-16 RNAi animals start with more progenitors on D1 and, therefore, are left with more germline progenitors on D12 than the RNAi control (see Supplementary Fig. 3). The decline between D1 and D12 is similar for 'germline' control and daf-16 RNAi. (c,d) In all cases, daf-16( + ) encodes DAF-16 isoform a1. Label on the bar indicates the tissue that expresses the transgene. Error bar indicates s.e.m.; NS, $P>0.05$; ${ }^{\star \star} P<0.01 ;{ }^{\star \star \star} P<0.001 ;{ }^{\star \star \star \star} P<0.0001$ by two-tailed Student's $t$-test. Alleles used: daf-2(e1370), daf-16(mu86) (in a,c), daf-16(m26) (in d), rrf-1(pk1417) and rde-1(ne219). See Supplementary Table 3 for complete genotypes and data. NS, not significant.

DAF-16/FOXO is required in this region suggested the possibility that germ cell flux through the proximal reproductive tract might influence the distal germline progenitor cell pool. We took advantage of $C$. elegans reproductive biology to test this hypothesis. The hermaphrodite reproductive system is essentially female except that $\sim 300$ self-sperm are produced before exclusive oocyte production ${ }^{22}$. Since hermaphrodites produce oocytes in excess, the number of self-progeny produced by unmated hermaphrodites is limited by the number of self-sperm, and reproduction ends when self-sperm are depleted. By contrast, when provided with additional sperm through mating, hermaphrodites can produce as many as 1,000 progeny and their reproductive span is extended ${ }^{23}$.

We, therefore, tested the hypothesis that germ cell flux might influence the distal germline progenitor pool by manipulating sperm availability. First, we examined $f \circ g-2(-)$ females that produce no self-sperm. These animals ovulate at a reduced rate due to the lack of the major sperm protein oocyte maturation signal from sperm, and, thus, have a much slower flux of germ cells through their reproductive tract. As a result, un-ovulated immature oocytes stack up in the oviduct ${ }^{24}$. We found that fog$2(-)$ females contained many more germline progenitor cells than wild-type hermaphrodites on D12 (Fig. 3a), suggesting that blocking germ cell flux inhibits the loss of germline progenitors over time. To test whether increasing germ cell flux would have the opposite effect, we mated young, wild-type adult males with wild-type hermaphrodites or fog-2(-) females on D4, when wild-type hermaphrodites have almost exhausted their selfsperm $^{12}$. By D6, both mated wild-type hermaphrodites and fog$2(-)$ females had fewer germline progenitors relative to their unmated controls, respectively (Fig. 3b), again supporting the notion that germ cell flux promotes germline progenitor cell loss and that germline progenitors are essentially 'used up' over time.

To further investigate a correlation between germline progenitor loss and germ cell flux, we examined two additional mutants, acy-4(-) and lin-3(-), in which germ cell flux is blocked in the 
a

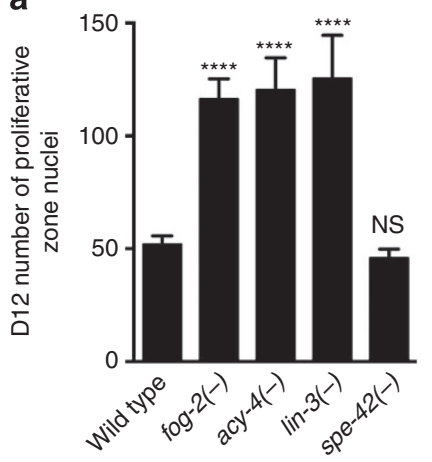

b

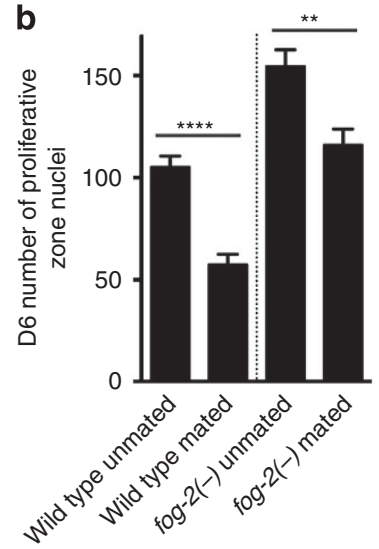

Figure 3 | Germ cell flux promotes germline progenitor loss. Average number of proliferative zone nuclei in (a) D12 wild-type, fog-2(-), acy-4( - ), lin-3( - ) and spe-42( - ) animals, (b) D6 wild type and fog-2( - ) mutants that are unmated or have been mated with young, wild-type adult males since D4. Error bar indicates s.e.m.; NS, $P>0.05$; ${ }^{\star \star} P<0.01$; ${ }^{\star \star \star} P<0.0001$ by two-tailed Student's $t$-test. Alleles used are as follows: fog-2(oz40), acy-4(ok1806), lin-3(n1058) and spe-42(tn1231). See Supplementary Table 3 for complete genotypes and data.

presence of self-sperm. These mutations interfere with oocyte maturation and ovulation, respectively ${ }^{25,26}$. We found that similar to fog-2(-), germline progenitor cell loss is inhibited in animals bearing either of these two mutations (Fig. 3a). Because reducing germ cell flux inhibits germline progenitor cell loss independent of the presence of sperm, our results suggest that germ cell flux, rather than sperm availability, influences germline progenitor maintenance over time.

To rule out a role for fertilization and progeny production in germline progenitor maintenance, we tested a fertilizationdefective mutant, spe-42(-). In this mutant, sperm are present and produce major sperm protein that promotes oocyte meiotic maturation, but these sperm are incapable of fertilizing oocytes. As a result, germ cell flux is normal (Supplementary Fig. 6a) but unfertilized oocytes accumulate in the uterus and are eventually expelled from the body; no viable progeny are produced ${ }^{27}$. We found that germline progenitors in this mutant were lost similarly to those in wild type (Fig. 3a). Taken together, our mutant analysis suggests that germ cell flux, rather than sperm, fertilization or viable progeny production promotes germline progenitor cell loss over time.

Germ cell flux acts partly through DAF-16/FOXO. Since either blocking germ cell flux or reducing daf-2 activity causes a similar phenotype in which old worms maintain a larger population of germline progenitors, and since the daf-2(rf) effect is suppressed by loss of $d a f-16$, we asked whether the maintenance of germline progenitors in response to reduced germ cell flux is also dependent on daf-16. We found that in the absence of daf-16, fog-2(-) animals lost more germline progenitors over time, although not to the same extent as the wild type (Fig. 4a, daf-16( - ); fog-2(-) versus fog-2(-) and wild type, respectively). This result suggests that daf-16(+) activity is required for the full effect of blocking germ cell flux to maintain germline progenitors. Importantly, loss of daf-16 did not restore germ cell flux in the fog-2(-) animals (Fig. 4b; Supplementary Fig. 6b), ruling out the possibility that the loss of germline progenitors seen in the daf-16(-); fog-2(-) double mutant is simply a result of resuming germ cell flux. Consistent with a major role for daf-16 in mediating the effect of
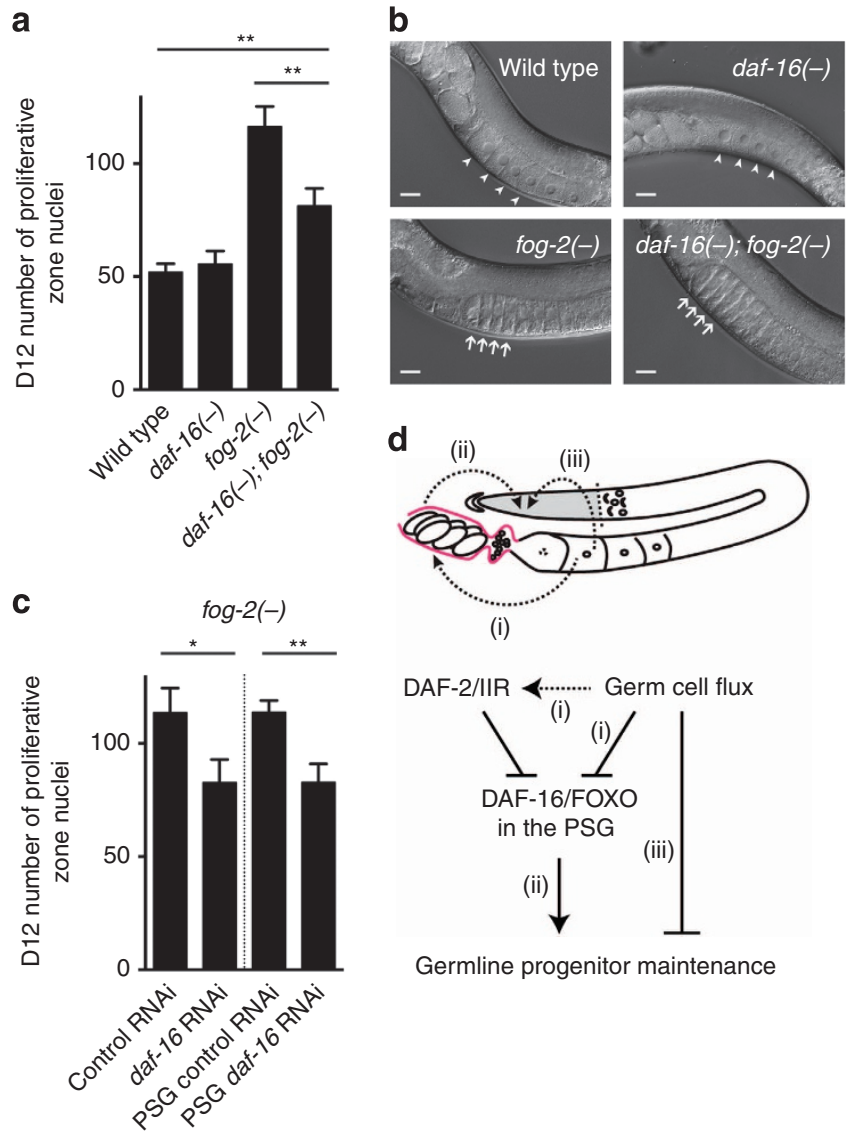

Germline progenitor maintenance

Figure 4 | Germ cell flux influences germline progenitor maintenance through DAF-16/FOXO-dependent and DAF-16/FOXO-independent mechanisms. (a) Average number of proliferative zone nuclei in D12 wildtype, daf-16( -$)$, fog-2( -$)$ and daf-16( -$)$; fog-2( -$)$ animals. (b) Representative DIC images of D1 wild-type, daf-16(-), fog-2(-) and daf16( - ); fog-2( -$)$ germ lines. Arrowhead: normal oocytes. Arrow: stacked oocytes. All daf-16( -$)$; fog-2(-) animals show the oocyte stacking phenotype $(n>50)$. Scale bars, $20 \mu \mathrm{m}$. (c) Average number of proliferative zone nuclei in D12 fog-2(-) and $r d e-1(-)$, fog-2( - ); Is [Pfos-1a::rde- $1(+)]$ animals treated with control and daf-16 RNAi. Error bar indicates s.e.m.; ${ }^{\star} P<0.05 ;{ }^{\star \star} P<0.01$ by two-tailed Student's $t$-test. Alleles used are as follows: fog-2(oz40), daf-16(mu86) and rde-1(ne219). See Supplementary Table 3 for complete genotypes and data. (d) Model. Pink line outlines the proximal somatic gonad. Areas for future inquiry are as follows: (i) links between germ cell flux and IIS (the effects of germ cell flux that are DAF16/FOXO dependent may occur through DAF-2/IIR, as indicated by dotted arrow); (ii) relevant DAF-16/FOXO targets in the proximal somatic gonad that signal to the distal stem/progenitor cell pool; and (iii) mechanism by which germ cell flux influences germline progenitors that is independent of proximal somatic gonad DAF-16/FOXO activity. DIC, differential interference contrast.

germ cell flux on germline progenitor maintenance, loss of daf-16 abolished the effect of mating (therefore, increasing germ cell flux) on the germline progenitor pool in the daf-2(rf) animals (Supplementary Fig. 7).

To determine whether daf-16(+) is also required in the proximal somatic gonad in this context, we performed tissuespecific daf-16 RNAi. We found that knocking down daf-16 in the proximal somatic gonad alone had the same effect as daf-16 RNAi in the whole animal: more pronounced progenitor loss in the fog-2(-) low-flux background (Fig. 4c). Together, these data suggest that germ cell flux influences germline progenitor 
maintenance through mechanisms that are dependent and independent of DAF-16/FOXO activity in the proximal somatic gonad.

\section{Discussion}

Our results show that (1) the stem/progenitor cell pool in the $C$. elegans germ line becomes depleted over time; (2) reducing IIS maintains this pool through DAF-16/FOXO activity in the proximal somatic gonad; and (3) blocking germ cell flux also maintains germline progenitors and this effect partially depends on proximal somatic gonad DAF-16/FOXO activity (Fig. 4d).

Our results offer three conceptual implications that may be generally relevant to age-related behaviour of stem cells. First, we demonstrate that the extent of germline progenitor cell loss over time can be uncoupled from the rate of organismal aging: intestinal daf-16(+) activity extends lifespan, but has no effect on age-dependent germline progenitor cell loss, while proximal somatic gonad daf-16(+) activity maintains germline progenitors without having much effect on lifespan. These results indicate that even though germline progenitor maintenance over time and lifespan are both regulated by IIS, they are independent phenotypes and DAF-16/FOXO regulates these two processes separately. We speculate that in other species, aging of tissue stem cells and organismal aging may similarly share molecular pathways but diverge in terms of regulative cell autonomy.

Although our results demonstrate distinct anatomical regulation of germline progenitor maintenance and lifespan at the level of DAF-16/FOXO activity, they may be linked upstream since both respond to DAF-2/IIR. From an evolutionary perspective, it is noteworthy that DAF-2 levels may thereby have a coordinate effect on reproduction and lifespan. For example, reducing daf-2 activity both extends lifespan and maintains germline progenitors, the combination of which could conceivably coordinately extend reproductive span and lifespan.

Second, our results imply that maintenance of tissue stem cells is not necessarily prioritized. In contrast to a scenario where a tissue stem cell population is positively regulated by maturation of the relevant progeny cells (thereby maintaining progenitor cell numbers), we show that under laboratory conditions, germline progenitors in C. elegans are 'used up'. That is, blocking germ cell flux maintains germline progenitors, while increasing germ cell flux exacerbates the loss of germline progenitors over time. This interpretation is consistent with the reduction in germline progenitors seen in wild-type animals mated over D1-D4 (ref. 28). We speculate that in this relatively short-lived species, maintenance of a large population of germline progenitors is not necessary as reproduction progresses. This idea is compatible with an evolutionary prioritization of an optimal, rather than maximal, number of progeny suggested previously by Hughes et al. ${ }^{12}$. Maintenance of other types of tissue stem cells may not be prioritized in other contexts where their maintenance may have been evolutionarily costly.

Finally, our data show that maintenance of the distal stem/ progenitor cell pool can be influenced by germ cell flux or IIS output from somatic cells at the proximal end of the reproductive tract. Importantly, this proximal area contacts mature gametes and embryos that were derived from the distal stem/progenitor cell pool. Thus, in addition to signals from the local niche ${ }^{2}$ and the systemic environment ${ }^{29}$, stem/progenitor cells can be regulated at the tissue/organ level. Similar mechanisms may operate in other tissue stem cell systems.

So how could $C$. elegans maintain germline progenitors when germ cell flux is reduced? We speculate that a product generated under conditions of reduced flux, for example, from stacked oocytes or germ cells undergoing a delay in meiotic progression ${ }^{30}$, may relay a signal to the distal germ line that retains germline progenitors until, for example, the hermaphrodite mates and can resume active reproduction.

Our studies also raise interesting questions for future enquiry such as the precise links between germ cell flux and IIS in regulating germline progenitor maintenance, the targets of DAF-16/FOXO in the proximal somatic gonad that signal to the distal stem/progenitor cell pool, and the mechanism by which germ cell flux influences germline progenitors that is independent of proximal somatic gonad DAF-16/FOXO activity (Fig. 4d).

\section{Methods}

Strains and plasmids. Strains were derived from N2 wild type (Bristol) and handled using standard methods ${ }^{31}$. Unless otherwise indicated, worms were grown on OP50 at $20^{\circ} \mathrm{C}$. Strains and plasmids constructed for this study are as follows: GC1332 daf-16(mu86); daf-2(e1370), GC1285 daf-16(m26); daf-2(e1370); naEx239[pGC629(Pfos-1a::gfp::daf-16) + pRF4], GC1337 rde-1(ne219); qyIs102[Pfos-1a::rde-1(genomic) + myo-2::yfp + unc-119(+)], GC1352 daf2(e1370); rde-1(ne219); qyIs102[Pfos-1a::rde-1(genomic) + myo-2::yfp + unc$119(+)$ ], GC1335 daf-16(mu86); fog-2(oz40), GC1353 rde-1(ne219), fog-2(oz40); qyIs102[Pfos-1a::rde-1(genomic) + myo-2::yfp + unc-119(+)] and pGC629 Pfosla::gfp::daf-16. Full genotypes of strains used are in Supplementary Table 1. Construction details for strains and plasmids are in Supplementary Tables 1 and 2, respectively.

Analysis of germline progenitor cells. Synchronization by L1 hatch-off, ethanol fixation and 4,6-diamidino-2-phenylindole staining were performed as described ${ }^{32}$. For hatch-off synchronization, all larvae and adults were washed off the bacterial lawn of plates of mixed-stage worms with M9 buffer. The remaining embryos were allowed to hatch for $2 \mathrm{~h}$ at $20^{\circ} \mathrm{C}$. Newly hatched L1 larvae were collected, distributed to plates seeded with OP50 or RNAi bacteria and grown at $20^{\circ} \mathrm{C}$. Worms were then staged by vulval development (see Fig. 4 of ref. 33), transferred onto fresh plates every other day until reproduction cessation and fixed at 1, 6 and 12 days post-L4 stage. For fixation and staining, worms were washed off plates with M9 buffer, fixed in $100 \%$ ethanol for 5-10 min and stained using VECTASHIELD mounting medium with 4,6-diamidino-2-phenylindole (Vector Laboratories, Burlingame, CA, USA).

Microscopy and determination of number of nuclei in the proliferative zone, distance to meiotic entry and mitotic index were as described ${ }^{13}$. All images were acquired with a Zeiss Imager Z1 equipped with an Apotome Axioimager (Carl Zeiss). The number of proliferative zone nuclei included all the germ cell nuclei between the distal tip and the beginning of meiotic entry, defined as the first row of cells in which two or more nuclei displayed the characteristic crescent shape. Distance to meiotic entry was measured in cell diameters from the distal tip to the meiotic entry border. The mitotic index was determined as the percentage of metaphase and anaphase figures over the total number of proliferative zone nuclei.

RNAi. RNAi by bacterial feeding was performed as described ${ }^{34}$ : worms were synchronized by L1 hatch-off (as described above), put onto plates with HT115 carrying control (L4440) or experimental plasmid and transferred onto fresh plates every other day until reproduction cessation at $20^{\circ} \mathrm{C}$. All RNAi results were pooled from at least two independent experiments.

Lifespan analysis. Lifespan experiments were performed at $20^{\circ} \mathrm{C}$ as described ${ }^{35}$ : $\sim 100 \mathrm{~L} 4\left(t_{0}\right)$ larvae were picked and transferred onto fresh plates every other day until reproduction cessation. Worms that crawled off the plate or died as a result of extruded internal organs ('bursting') or internally hatched progeny ('bagging') were censored but were included in the statistical analysis as censored animals. Survival curves were generated using Prism 6.

Mating. Mating experiments were performed as described ${ }^{12}$ : day 4 adult wild-type hermaphrodites or fog-2(oz40) females were mated with three young, wild-type adult males individually for 2 days. Mating was later confirmed by the presence of male progeny.

Quantitation of ovulation rate. Ovulation rate of D1 animals were determined as in ref. 36. First, worms were mounted in M9 for Nomarski microscopy and the number of oocytes and embryos in the spermathecae and uterus of each animal was determined. Animals were then transferred individually to fresh plates with OP50 for 2-3 h. At the end of the time interval, oocytes and embryos in the spermathecae and uterus were counted again. Embryos or unfertilized oocytes laid 
on the plate were also counted. The ovulation rate per gonad arm per hour was then calculated.

\section{References}

1. Signer, R. A. J. \& Morrison, S. J. Mechanisms that regulate stem cell aging and life span. Cell Stem Cell 12, 152-165 (2013).

2. Kershner, A. et al. Germline stem cells and their regulation in the nematode Caenorhabditis elegans. Adv. Exp. Med. Biol. 786, 29-46 (2013).

3. Morrison, S. J. \& Spradling, A. C. Stem cells and niches: mechanisms that promote stem cell maintenance throughout life. Cell 132, 598-611 (2008).

4. Kenyon, C. J. The genetics of ageing. Nature 464, 504-512 (2010).

5. Killian, D. J. \& Hubbard, E. J. A. Caenorhabditis elegans germline patterning requires coordinated development of the somatic gonadal sheath and the germ line. Dev. Biol. 279, 322-335 (2005).

6. Garigan, D. et al. Genetic analysis of tissue aging in Caenorhabditis elegans: a role for heat-shock factor and bacterial proliferation. Genetics 161, 1101-1112 (2002).

7. Luo, S., Kleemann, G. A., Ashraf, J. M., Shaw, W. M. \& Murphy, C. T. TGF- $\beta$ and insulin signaling regulate reproductive aging via oocyte and germline quality maintenance. Cell 143, 299-312 (2010).

8. Gumienny, T. L., Lambie, E., Hartwieg, E., Horvitz, H. R. \& Hengartner, M. O. Genetic control of programmed cell death in the Caenorhabditis elegans hermaphrodite germline. Development 126, 1011-1022 (1999).

9. Hansen, D. \& Schedl, T. Stem cell proliferation versus meiotic fate decision in Caenorhabditis elegans. Adv. Exp. Med. Biol. 757, 71-99 (2013).

10. Kenyon, C., Chang, J., Gensch, E., Rudner, A. \& Tabtiang, R. A C. elegans mutant that lives twice as long as wild type. Nature 366, 461-464 (1993).

11. Gems, D. et al. Two pleiotropic classes of daf-2 mutation affect larval arrest, adult behavior, reproduction and longevity in Caenorhabditis elegans. Genetics 150, 129-155 (1998).

12. Hughes, S. E., Evason, K., Xiong, C. \& Kornfeld, K. Genetic and pharmacological factors that influence reproductive aging in nematodes. PLoS Genet. 3, e25 (2007).

13. Michaelson, D., Korta, D. Z., Capua, Y. \& Hubbard, E. J. A. Insulin signaling promotes germline proliferation in C. elegans. Development 137, 671-680 (2010).

14. Murphy, C. T. \& Hu, P. J. In WormBook: The Online Review of C. elegans Biology. (Internet) 1-43 (WormBook, 2013).

15. Sijen, T. et al. On the role of RNA amplification in dsRNA-triggered gene silencing. Cell 107, 465-476 (2001).

16. Kumsta, C. \& Hansen, M. C. elegans rrf-1 mutations maintain RNAi efficiency in the soma in addition to the germline. PLoS ONE 7, e35428 (2012).

17. Sherwood, D. R., Butler, J. A., Kramer, J. M. \& Sternberg, P. W. FOS-1 promotes basement-membrane removal during anchor-cell invasion in $C$. elegans. Cell 121, 951-962 (2005).

18. Libina, N., Berman, J. R. \& Kenyon, C. Tissue-specific activities of C. elegans DAF-16 in the regulation of lifespan. Cell 115, 489-502 (2003).

19. Hagedorn, E. J. et al. Integrin acts upstream of netrin signaling to regulate formation of the anchor cell's invasive membrane in C. elegans. Dev. Cell 17, 187-198 (2009).

20. Zhang, P., Judy, M., Lee, S.-J. \& Kenyon, C. Direct and indirect gene regulation by a life-extending FOXO protein in C. elegans: roles for GATA factors and lipid gene regulators. Cell Metab. 17, 85-100 (2013).

21. Qadota, H. et al. Establishment of a tissue-specific RNAi system in C. elegans. Gene 400, 166-173 (2007).

22. Zanetti, S. \& Puoti, A. Sex determination in the Caenorhabditis elegans germline. Adv. Exp. Med. Biol. 757, 41-69 (2013).

23. Marcello, M. R., Singaravelu, G. \& Singson, A. Fertilization. Adv. Exp. Med. Biol. 757, 321-350 (2013).

24. Kim, S., Spike, C. \& Greenstein, D. Control of oocyte growth and meiotic maturation in Caenorhabditis elegans. Adv. Exp. Med. Biol. 757, 277-320 (2013).

25. Govindan, J. A., Nadarajan, S., Kim, S., Starich, T. A. \& Greenstein, D. Somatic cAMP signaling regulates MSP-dependent oocyte growth and meiotic maturation in C. elegans. Development 136, 2211-2221 (2009).
26. Clandinin, T. R., DeModena, J. A. \& Sternberg, P. W. Inositol trisphosphate mediates a RAS-independent response to LET-23 receptor tyrosine kinase activation in C. elegans. Cell 92, 523-533 (1998).

27. Kroft, T. L., Gleason, E. J. \& L'Hernault, S. W. The spe-42 gene is required for sperm-egg interactions during C. elegans fertilization and encodes a sperm-specific transmembrane protein. Dev. Biol. 286, 169-181 (2005).

28. Shi, C. \& Murphy, C. T. Mating induces shrinking and death in Caenorhabditis mothers. Science 343, 536-540 (2014).

29. Hubbard, E. J. A., Korta, D. Z. \& Dalfó, D. Physiological control of germline development. Adv. Exp. Med. Biol. 757, 101-131 (2013).

30. Jaramillo-Lambert, A., Ellefson, M., Villeneuve, A. M. \& Engebrecht, J. Differential timing of $\mathrm{S}$ phases, $\mathrm{X}$ chromosome replication, and meiotic prophase in the C. elegans germ line. Dev. Biol. 308, 206-221 (2007).

31. Brenner, S. The genetics of Caenorhabditis elegans. Genetics 77, 71-94 (1974).

32. Pepper, A. S.-R., Killian, D. J. \& Hubbard, E. J. A. Genetic analysis of Caenorhabditis elegans glp-1 mutants suggests receptor interaction or competition. Genetics 163, 115-132 (2003).

33. Seydoux, G., Savage, C. \& Greenwald, I. Isolation and characterization of mutations causing abnormal eversion of the vulva in Caenorhabditis elegans. Dev. Biol. 157, 423-436 (1993).

34. Timmons, L., Court, D. L. \& Fire, A. Ingestion of bacterially expressed dsRNAs can produce specific and potent genetic interference in Caenorhabditis elegans. Gene 263, 103-112 (2001).

35. Tank, E. M. H., Rodgers, K. E. \& Kenyon, C. Spontaneous age-related neurite branching in Caenorhabditis elegans. J. Neurosci. 31, 9279-9288 (2011).

36. McCarter, J., Bartlett, B., Dang, T. \& Schedl, T. On the control of oocyte meiotic maturation and ovulation in Caenorhabditis elegans. Dev. Biol. 205, 111-128 (1999).

37. Crittenden, S. L., Leonhard, K. A., Byrd, D. T. \& Kimble, J. Cellular analyses of the mitotic region in the Caenorhabditis elegans adult germ line. Mol. Biol. Cell 17, 3051-3061 (2006).

\section{Acknowledgements}

We thank David Sherwood, Cynthia Kenyon, David Greenstein, Andy Singson, Jeremy Nance, Niels Ringstad and Ruth Lehmann for reagents and/or advice, Don Ryoo and Mayumi Ito for comments on the manuscript, Michelle LeBlanc for initial observations and Yan Deng at the NYULMC Microscopy core for assistance with microscopy (NCRR S10 RR024708). Some strains were provided by the CGC, which is funded by NIH Office of Research Infrastructure Programs (P40 OD010440). This work was supported by NIH R03AG042551, R03AG042551-S1 and R01GM061706, R01GM061706-S1.

\section{Author contributions}

Z.Q. and E.J.A.H. designed the research; Z.Q. performed the experiments; Z.Q. analysed the data; and Z.Q. and E.J.A.H. wrote the manuscript.

\section{Additional information}

Supplementary Information accompanies this paper at http://www.nature.com/ naturecommunications

Competing financial interests: The authors declare no competing financial interests.

Reprints and permission information is available online at http://npg.nature.com/ reprintsandpermissions/

How to cite this article: Zhao Qin \& E. Jane Albert Hubbard, Non-autonomous DAF16/FOXO activity antagonizes age-related loss of C. elegans germline stem/progenitor cells. Nat. Commun. 6:7107 doi: 10.1038/ncomms8107 (2015).

This work is licensed under a Creative Commons Attribution 4.0 International License. The images or other third party material in this article are included in the article's Creative Commons license, unless indicated otherwise in the credit line; if the material is not included under the Creative Commons license, users will need to obtain permission from the license holder to reproduce the material. To view a copy of this license, visit http://creativecommons.org/licenses/by/4.0/ 